\title{
Bulges and ellipticals: can formation mechanisms be the same?
}

\author{
S.N. NURITDINOV \\ Tashkent University, Physical Faculty, Astronomy Department, Tashkent, 700095, \\ Republic Uzbekistan
}

We consider three mechanisms for ellipticals and bulge formation.

1. Mechanisms within a dissipationless collapse scenario: for example, the radialorbits instability (for non-stationary models see V.A. Antonov \& S.N. Nuritdinov, 1981, Sov. Astr. Zh., 58, 1158; L. Aguilar \& D. Merrit, 1990, Ap. J., 345, 33; Nuritdinov, this issue).

2. Evolution of (proto)galaxy from an anisotropic sphere or a spheroidal model (A.M. Fridman \& V.L. Polyachenko, 1984, Physics of gravitating systems, Springer). Here axisymmetric oscillations $(m=0, N=4)$ correspond to the case of a galaxy with a bulge, and the ellipsoidal mode $(m=N=2)$ corresponds to the case of ellipticals. In principle these models can have a halo or a corona with a given mass (Nuritdinov, 1978, Sov. Astr. Zh., 55, 37).

3. Dissipation phenomena in non-stationary models.

Now we proceed to analyse the role of the "dome" instability during non-stationary evolution in order to check a relation of this instability to the bulge formation problem. Nuritdinov (1987, Dinamica gravitiruyshchih system i metodi analyt. neb. meh., p65) has constructed two phase models of pulsating disk. One of these models is

$$
\Psi=\frac{\sigma_{0}}{2 \pi \Pi \sqrt{1-\Omega^{2}}}\left[\frac{1-\Omega^{2}}{\Pi^{4}}\left(\Pi^{2}-r^{2}\right)-\left(v_{r}-v_{a}\right)^{2}-\left(v_{\perp}-\frac{\Omega r}{\Pi^{2}}\right)^{2}\right]^{-\frac{1}{2}}
$$

where all notations are according Nuritdinov (this issue). Recently we have studied warps in this disk, assuming vertical displacements of the form $B(t) \frac{1}{\xi} P_{N}^{m}(\xi) e^{i m \varphi}$, where $B$ is a time function and $\xi=\sqrt{1-r^{2} / \Pi^{2}}$. Here we suffice to give the result for the dome perturbation $(m=0, N=3)$ : the stability region in the $(2 T /|u|, \Omega)$ plane (see figure) shows some interesting narrow channels. Moreover, we have calculated the unstable modes as a function of $2 T /|\mathcal{U}|$ and $\Omega$. Our analysis shows that the dome instability can play a role in the formation of bulges.

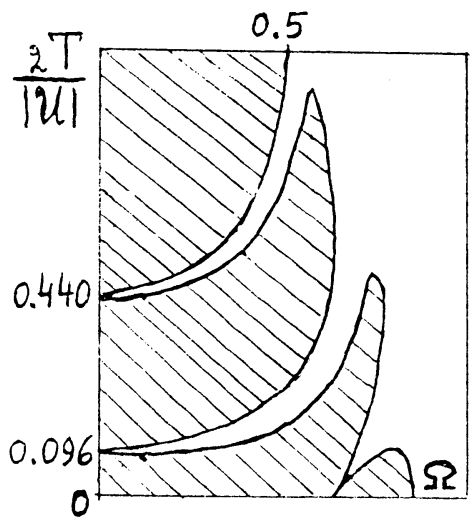




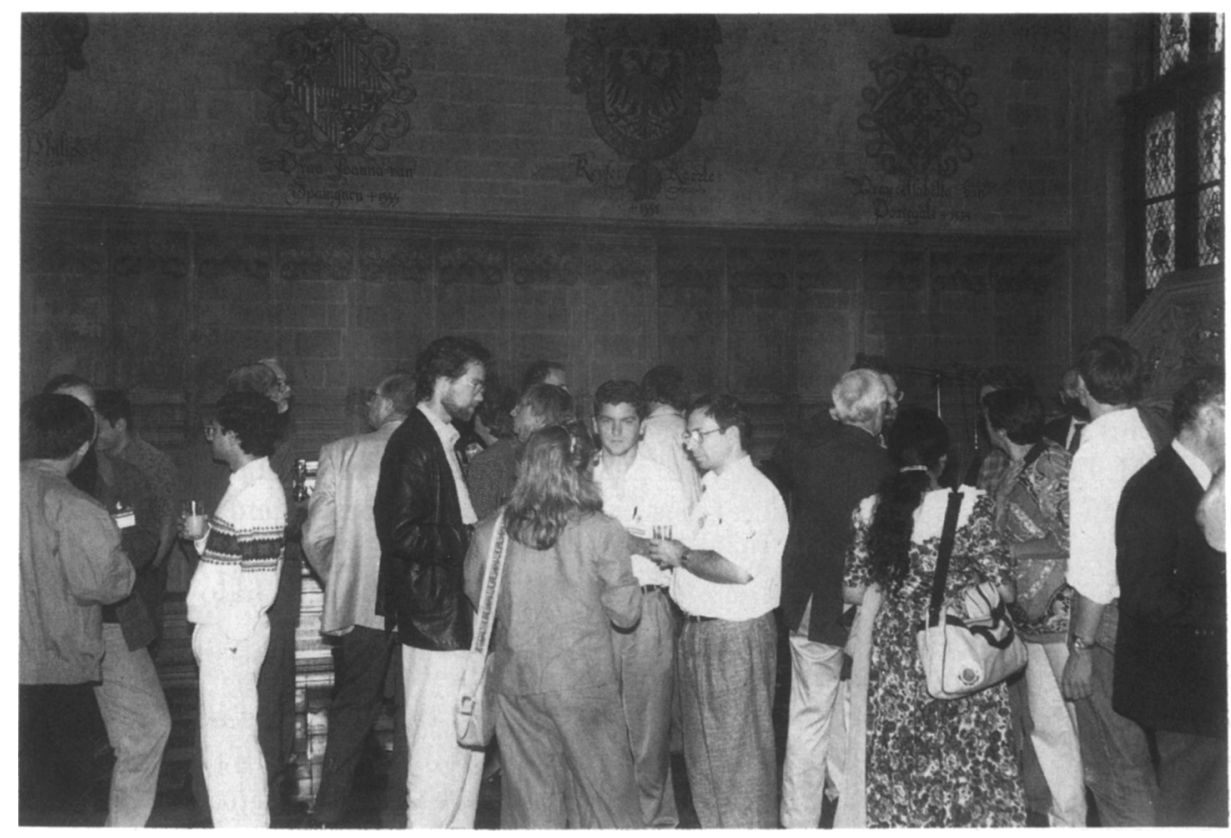

Reception at the town hall

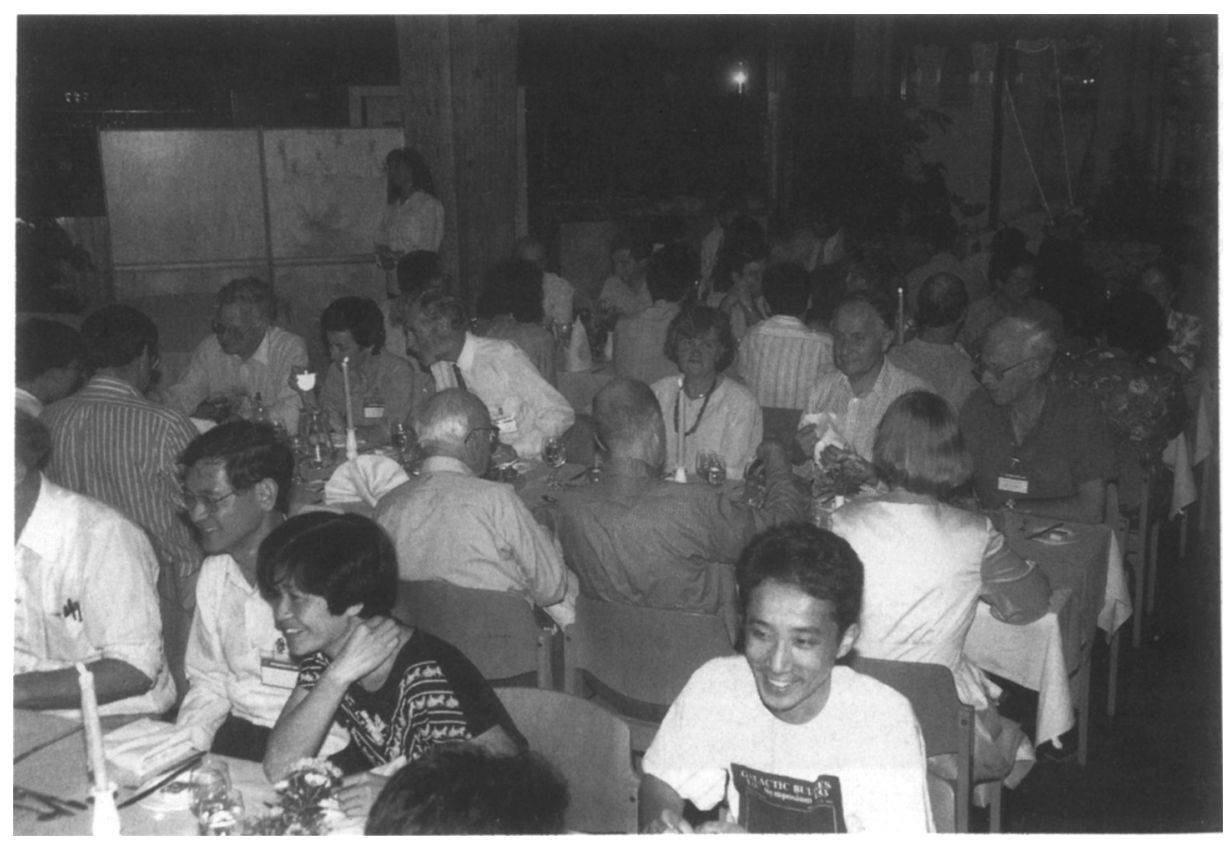

The conference dinner 